\title{
Variables asociadas a la satisfacción del paciente en una unidad de hemodiálisis
}

\author{
Mercedes Miguel Montoya* - Covadonga Valdés Arias** - Manuela Rábano Colino* - Yolanda Artos \\ Montes* - Pilar Cabello Valle* - Natividad De Castro Prieto* - Ana García León* - Azucena Martínez \\ Villoria* - Francisco Ortega Suárez***
}

*Diplomada en Enfermería - * *icenciada en Psicología - * * Doctor en Medicina

Unidad de Hemodiálisis. Servicio de Nefrología. Hospital Universitario Central de Asturias.

Red de Investigación en Enfermedades Renales

\section{Resumen}

Introducción. Todos los planes de calidad en el ámbito hospitalario tienen en cuenta la satisfacción del paciente como uno de los principales objetivos a conseguir. Las medidas de los resultados obtenidos de las opiniones de los pacientes permiten tomar decisiones que mejoran la calidad de los cuidados.

Objetivos. Evaluar la Satisfacción de los pacientes de una Unidad de Hemodiálisis, y analizar los aspectos, tanto del paciente como de la enfermedad, que pudieran condicionarla.

Material y métodos. Los pacientes fueron entrevistados con el Cuestionario SERVQHOS modificado para hemodiálisis, que evalúa la Satisfacción con veinte atributos de la Unidad y con el cuestionario de Calidad de Vida Relacionada con la Salud, EUROQOL. También se recogieron las respuestas a cuestiones que podrían condicionar la satisfacción, variables clínicas y sociodemográficas.

Resultados. Completaron la entrevista 50 pacientes y los niveles de satisfacción fueron altos y similares a los de otras unidades en España. Menores niveles de satisfacción se asociaron a percibir más

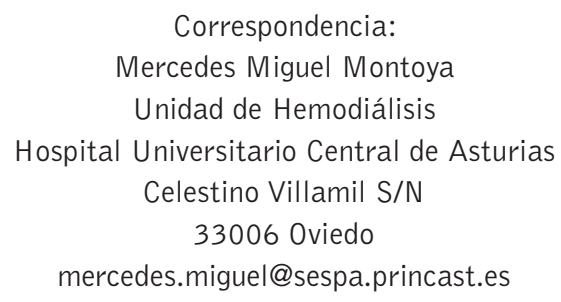

Correspondencia:

Mercedes Miguel Montoya

Unidad de Hemodiálisis

Hospital Universitario Central de Asturias

Celestino Villamil S/N

33006 Oviedo

mercedes.miguel@sespa.princast.es

Dolor, más limitación para las Actividades Cotidianas, más Ansiedad/Depresión, Ilevar más tiempo en diálisis, más horas de sesión, dializarse por catéter, haber estado trasplantado y tener menor edad $(p<0,05)$. Por el contrario, menor nivel de estudios, actividades laborales menos cualificadas, ir en ambulancia, haber elegido modalidad de diálisis, y considerar que su opinión es tenida en cuenta, aumentaban la satisfacción $(p<0,05)$.

Conclusiones. Identificar los factores que modulan la satisfacción es tan importante como evaluarla, además deben de tenerse en cuenta las variables inherentes del paciente, que están condicionando la satisfacción percibida.

\section{PALABRAS CLAVE:}

- HEMODIÁLISIS

- SATISFACCIÓN DEL PACIENTE

- CALIDAD DE VIDA

\section{Variables associated to patient satisfaction in a haemodialysis unit}

\section{Abstract}

Introduction. All quality plans in hospitals take patient satisfaction into account as one of the main goals to achieve. The measurements of the results obtained from patients' opinions allow decisions to be made that improve the quality of care.

Objectives. To evaluate the satisfaction of patients of a haemodialysis unit, and analyse the aspects, 
both of the patient and of the disease, that could be conditioning factors.

Material and methods. Patients were interviewed with the SERVQHOS questionnaire modified for haemodialysis, which evaluates satisfaction with twenty attributes of the unit and with the EUROQOL health-related quality of life questionnaire. The replies to questions that could affect satisfaction, clinical and sociodemographic variables were also noted.

Results. The interview was completed by 50 patients and the levels of satisfaction were high, and similar to those of other units in Spain. Lower levels of satisfaction were associated with feeling more pain, more limitations for daily activities, more anxiety/depression, having been on dialysis longer, longer sessions, receiving dialysis using a catheter, having had a transplant and being younger $(p<0.05)$. On the other hand, a lower level of qualifications, less qualified work, travelling by ambulance, having chosen the form of dialysis and considering that their opinion is taken into accounts increased satisfaction $(p<0.05)$.

Conclusions. Identifying the factors that modulate satisfaction is as important as evaluating it. The inherent variable of the patient must also be taken into consideration, as they condition the perceived satisfaction.

\section{KEY WORDS:}

HAEMODIALYSIS

PATIENT SATISFACTION

QUALITY OF LIFE

\section{Introducción}

El aumento de la esperanza de vida genera poblaciones cada vez más ancianas y con mayores necesidades y demandas. También conlleva un aumento del número de pacientes con enfermedades crónicas que les convierte en usuarios de los servicios sanitarios de por vida. En este contexto, las políticas sanitarias no se pueden desarrollar sin tener en cuenta su opinión y sin considerar los cambios que suponen un valor añadido para la percepción de su salud o bienestar. El concepto de calidad va surgiendo con más fuerza en los diferentes campos de la gestión en general y en la gestión sanitaria y asistencial en particular. El usuario, el cliente o el ciudadano, exige más prestaciones y de mejor calidad, especialmente en los servicios dirigidos a las personas, como el de la sanidad. Parejo al concepto de calidad ha hecho su aparición la importancia de hacer su evaluación y las instituciones de salud no han estado ajenas a ello.

Todos los modelos de gestión de la calidad en salud, desde el Ciclo evaluativo o de Mejora de Deming, hasta las normas ISO-9000, el sistema de acreditación de la $J C A H O$ o el modelo europeo de excelencia de la $E F Q M$, se basan en la evaluación continua para conseguir un producto de calidad y satisfactorio para los clientes. A su vez una de las similitudes en los cuatro modelos es la importancia de la orientación hacia el cliente (el paciente), que todos destacan como característica primordial y que debe basarse en información contrastada acerca de las preferencias, las expectativas, lo que se considera relevante en cada momento, y tendencias en la opinión de los pacientes para enfocar la organización hacia las necesidades cambiantes de los usuarios ${ }^{1}$.

Los planes de calidad, al tener en cuenta la satisfacción del paciente como uno de los principales objetivos a conseguir, preconizan un mayor protagonismo de éstos y desarrollan un nuevo tipo de relaciones médico-paciente, evaluando los resultados de la atención sanitarias desde la perspectiva del paciente. El ciclo de la satisfacción del cliente consistiría en conocer sus necesidades, identificar las expectativas, establecer objetivos concretos, cuantificar y analizar los resultados, traducirlos al lenguaje interno y hacerlos presentes en toda la empresa, desplegando los objetivos a todos los niveles. Por otro lado, parece quedar inherente al concepto de satisfacción, el concepto de expectativa y es que el grado de satisfacción no depende sólo de la prestación de los servicios, sino también de la solución de sus expectativas, ya que el paciente tendrá más satisfacción cuando dichos servicios reúnan o sobrepasen las características que esperaban ${ }^{2}$. Cuando la expectativa del paciente es baja o está acostumbrado a tener un acceso limitado a los servicios, puede no percatarse del nivel real de calidad y estar recibiendo servicios deficientes sin ninguna objeción, lo que también puede influir en las tasas de insatisfacción del $3-5 \%$ en la sanidad pública, donde el paciente tiene 
una expectativa más baja que en los centros sanitarios privados. El método más utilizado para la medida son las encuestas de opinión, que permiten detectar aspectos susceptibles de mejora, insuficiencias o disconformidades y así elevar la calidad de los servicios para conseguir la excelencia clínica y la satisfacción de las expectativas de los ciudadanos.

En relación a los factores que determinan la satisfacción de los pacientes parecen importantes aquellos que tienen que ver con la calidad de la información que reciben sobre su enfermedad, sobre todo la relacionada con los riesgos del diagnóstico y del tratamiento, seguido de las instrucciones a seguir tras el alta de un ingreso y la información de las pruebas diagnósticas realizadas ${ }^{3}$. Levinson también afirma que la información detallada, aunque asequible, principalmente de los riesgos y su aceptación por parte del paciente, es un aspecto importante en la relación entre el médico y el paciente ${ }^{4,5}$. El conocimiento del nombre de los profesionales sanitarios, el tiempo dedicado a la consulta, la espera previa a la consulta y las dificultades para la citación son las oportunidades de mejora más expresadas de manera mayoritaria en el apartado de sugerencias de un estudio ${ }^{5}$.

No hay muchos estudios llevados a cabo hasta la fecha, sobre la satisfacción de los pacientes en las unidades de hemodiálisis (HD). En España, Hernández y Cols. ${ }^{6}$ en su estudio descriptivo transversal con el cuestionario SERVQHOS modificado, en su unidad con 60 pacientes capacitados para responder encuentran que los aspectos con los que los pacientes están menos satisfechos son los tiempos de espera para ser atendidos por un médico, la facilidad para llegar a la unidad, y la comunicación que el personal de la unidad mantiene con la familia. Por otro lado, vieron que los aspectos que más determinaban la satisfacción general con la unidad eran la puntualidad de las sesiones de hemodiálisis, la rapidez con la que conseguían lo que necesitaban, el interés del personal de enfermería por los pacientes y el tiempo de espera para ser atendido por un médico. No hallaron ninguna relación entre satisfacción y variables sociodemográficas. En un estudio muy reciente estos mismos autores comparan la satisfacción de los pacientes en hemodiálisis, que dependería de la atención que reciben y la de los pacientes en diálisis peritoneal, encontrando niveles más altos que los de hemodiálisis, que dependerían de la responsabilidad de poder elegir la técnica ${ }^{7}$. Otro estudio de Santos y Cols. en nuestro país, encuentran que la satisfacción general de los pacientes es alta, que la profesionalidad del personal sanitario es valorada positivamente, si bien los medios de transporte, están peor valorados, consecuencia del tiempo de espera y la accesibilidad. Estos autores tampoco encuentran relación entre satisfacción y variables sociodemográficas ${ }^{8}$.

Los objetivos de este trabajo fueron evaluar la satisfacción de los pacientes en la unidad de hemodiálisis y analizar los posibles factores, tanto del paciente como del tratamiento que pudieran estar asociados.

\section{Material y Métodos}

Todos los pacientes de la Unidad de HD del Hospital Universitario Central de Asturias que cumplían los criterios de inclusión fueron entrevistados por la psicóloga, perteneciente a la Unidad de Nefrología y entrenada en este tipo de cuestionarios, pero no encargada de sus cuidados para guardar la confidencialidad y evitar que los pacientes contestasen queriendo agradar, lo que podría producirse si hubiesen sido entrevistados por las enfermeras que se encargan de sus cuidados. Se siguieron las normas elementales de aplicación de este tipo de cuestionarios: privacidad, lectura de preguntas sin modificaciones, ni aclaraciones, etc. Se explicó la naturaleza y objetivos del estudio al paciente. Los pacientes en HD contestaron los cuestionarios en medio de la sesión. Los criterios de inclusión fueron: no estar ingresado en el momento del estudio, llevar como mínimo un mes en HD, poseer capacidad física y cognitiva para responder los cuestionarios y consentimiento del paciente.

Las entrevistas se llevaron a cabo en febrero de 2008 e incluyeron: para evaluar la satisfacción de los pacientes, el cuestionario SERVQHOS modificado para HD que anteriores estudios han utilizado en nuestro país. Es una adaptación para pacientes en hemodiálisis de la encuesta SERVQUAL (instrumento para evaluar la calidad percibida en empresas de servicios). Hernández y Cols. Io modificaron eliminando una pregunta que carecía de sentido en los pacientes en hemodiálisis y se incluyeron dos preguntas específicas relativas a la dieta y a la medicación. La consistencia interna en su estudio es de 0,95 , lo que indica que el instrumento de medida es fiable. Consta de 20 ítems que analizan los diferentes atributos que 
determinan la satisfacción de la asistencia sanitaria en función de las expectativas previas a la entrada a la Unidad de diálisis y que se puntúan con una escala de Likert, que va desde el 1 ("mucho peor de lo que esperaba") al 5 ("mucho mejor de lo que esperaba") 6 . Además se les preguntó (contestaban SI/NO) las siguientes cuestiones por su posible implicación con algunos aspectos de la satisfacción: si conocía el nombre del médico y de la supervisora; si creía que se le había explicado suficientemente la enfermedad y la terapia; si pudo elegir la terapia y el turno; si cree que su opinión es tenida en cuenta; si cree que está correctamente atendido en la unidad; si está satisfecho con el medio de transporte. Para evaluar su Calidad de Vida Relacionada con la Salud (CVRS) se usó el Cuestionario EUROQ0L o EQ-5D que mide la percepción del paciente en Movilidad, Cuidado Personal, Actividades Cotidianas, Dolor/Malestar y Ansiedad/Depresión. El paciente se valora en cada dimensión con 1-no tengo problemas, 2-tengo algunos problemas, 3-tengo muchos problemas. De la combinación a esas respuestas surge la Tarifa, o estado de salud general, que se transforma en un índice que va de 0-peor estado a 1- mejor estado. Por último, el paciente se valora a sí mismo de 0 -peor estado a 100-mejor estado, con una escala o Termómetro visual, que se le muestra9. En la entrevista con el pacientes también se recogieron las variables sociodemográficas: edad; sexo; estado civil (casado/a; viudo/a; soltero/a; separado/a); situación socioeconómica (nivel de ingresos de la unidad familiar); nivel de estudios completados (leer y escribir; primarios; bachiller/FP; superiores); situación laboral (trabaja a media jornada o a jornada completa; estudia; ama de casa; en paro; jubilado por enfermedad o por edad); composición del hogar (personas que comparten el domicilio en el momento actual).

En ese mismo día, una enfermera de la unidad evaluó a cada paciente con el índice de Barthel, que valora la capacidad de autocuidado. Consta de 10 ítems, cada uno de los cuales mide la dependencia o independencia, así como la continencia de esfínteres. La puntuación total de máxima independencia y de dependencia son 100 y 0 respectivamente ${ }^{10}$. También valoró el estado funcional con la escala de Karnofsky modificada, que es una escala objetiva para estimar limitaciones físicas, emocionales y mentales de los pacientes. Consta de once grados para situar al paciente según las limitaciones que tenga, siendo la puntuación mínima 45 (incapacitado por completo) y la máxima
100 (normal, sin evidencia de enfermedad) ${ }^{11}$. La enfermera recogió a su vez un listado de variables clínicas habituales en los estudios de pacientes renales en HD y las que se presupusieron que podían relacionarse con algún aspecto de la satisfacción: causa de Insuficiencia Renal Crónica Terminal (IRCT); tiempo previo en terapia sustitutiva renal (HD, DPCA, trasplante), parámetros de adecuación de diálisis (KtV y $\mathrm{KtVI}$ ); niveles de albúmina y hematocrito; índice de comorbilidad; medicaciones recibidas (número total de diferentes fármacos prescritos día); empleo de psicofármacos (hipnóticos, ansiolíticos y/o antidepresivos, situación en lista de trasplante).

\section{Análisis de los Datos}

Con el paquete estadístico SPSS 12.0 se procedió primero a introducir todas las respuestas de cada uno de los pacientes, se codificaron las variables y se realizó el estudio descriptivo de la muestra, utilizando la media y desviación estándar, para cada una de las variables cuantitativas y frecuencias absolutas y relativas para las categóricas. Se realizó el estudio analítico, buscando las relaciones de asociación entre las variables estudiadas y las puntuaciones en el instrumento de evaluación de la Satisfacción: como las puntuaciones del cuestionario para cada aspecto evaluado da una variable discreta (oscilan de 1 a 5) utilizamos la prueba no paramétrica "Coeficiente de Sperman" para valorar las asociaciones con otras variables cuantitativas discretas o continúas, y la prueba "t de Student" para comparar las puntuaciones entre dos muestras en las variables dicotómicas.

\section{Resultados}

En cuanto a las características sociodemográficas: De los 57 pacientes, se descartaron 3 pacientes por impedimentos físicos y 2 por cognitivos y 2 no desearon colaborar. Del resto, 50, que sí completaron la entrevista, se presentan las principales características sociodemográficas y clínicas en la tabla 1. La media de edad fue de 64,84 años, la mayor parte de los pacientes tuvieron niveles bajos de educación y estaban jubilados. La mayoría de ellos estaban en diálisis a consecuencia de Nefropatías vasculares o Diabetes. La media en tiempo de diálisis fue de 37,32 meses, aunque hubo una desviación de 46,63, lo que implica una gran dispersión de datos. El 72,4\% se dializaba a través de una fístula arteriovenosa, el 20\% estaba en lista para recibir un trasplante y los pacientes tu- 


\begin{tabular}{|c|c|}
\hline Edad & $64,84 \pm 17,64$ \\
\hline Sexo (\% varones) & $58 \%$ varones \\
\hline Situación de convivencia(\%familia) & $81,8 \%$ \\
\hline Nivel de educación bajo/elemental & $80 \%$ \\
\hline Incapacidad laboral & $91,8 \%$ \\
\hline Diagnóstico principal (\%) & $\begin{array}{l}30,4 \% \text { N.Vascular; } 23,2 \% \text { Diabetes mellitus; } 7,1 \% \text { Poliquistosis; } 8,9 \% \\
\text { Glomerulonefritis; } 3,6 \% \text { Nefropatía intersticial; } 5,4 \% \text { otros; } 21,4 \% \text { N/F }\end{array}$ \\
\hline Meses en hemodiálisis & $37,32 \pm 46,63$ \\
\hline Usa fístula para dializarse & $72,4 \%$ \\
\hline Horas de sesión (entre 3 y 4) & $87,6 \%$ \\
\hline Hematocrito \% & $35,68 \pm 5,40$ \\
\hline Hemoglobina (gr/dl) & $11,95 \pm 1,76$ \\
\hline Albúmina (gr/dl) & $3,6 \pm 0,41$ \\
\hline Está en lista de Trasplante & $20,8 \%$ \\
\hline $\mathrm{N}^{\circ}$ de fármacos diarios & $6,81 \pm 2$ \\
\hline $\mathrm{N}^{0}$ de enfermedades asociadas & $2,88 \pm 1,6$ \\
\hline
\end{tabular}

Tabla 1. Características sociodemográficas y clínicas de los pacientes

vieron una media de casi 3 enfermedades más asociadas a la enfermedad renal.

\section{En cuanto a la funcionalidad:}

En cuanto a la funcionalidad, medida por una enfermera de la unidad con la Escala de Karnofsky, se encontró que un $7 \%$ de los pacientes necesitaba ayuda para todo, un $16 \%$ necesitaba diferentes tipos de ayuda para su vida diaria y el $77 \%$ restante se valía por sí mismo. Con la Escala de Barthel, que evalúa dependencia, el $8,8 \%$ la tenía total o grave, el 8,8 $\%$ moderada y el 82,5 leve o no tenía.

\section{En cuanto a los Resultados en la Satisfacción:}

Las medias en las puntuaciones de los veinte atributos estuvieron todas entre 3,2 y 4,04 lo que indica que se encuentran entre como lo esperaba " 3 " y mejor de lo que esperaba "4". Los atributos mejor valorados fueron "la amabilidad del personal de la unidad" y "la disposición del personal", y los de menor, "la información que proporcionan los médicos" y la "información proporcionada sobre la dieta". Del análisis que se llevó a cabo para ver las variables que disminuían la satisfacción en esos veinte atributos (tabla 2), se encontró que a mayor tiempo en diálisis, tener sesiones de más horas, haber alcanzado mayor nivel de estudios, haber desarrollado una actividad laboral más cualificada, tener más limitaciones para realizar las actividades laborales, más dolor o malestar, o más ansiedad o depresión correlacionaron negativamente de una forma entre baja y moderada con alguno de los aspectos, disminuyendo la satisfacción de una forma estadísticamente significativa $(p<0,05)$.

\begin{tabular}{|c|c|c|c|c|c|c|c|}
\hline & $\begin{array}{l}\text { Mayor } \\
\text { tiempo } \\
\text { en HD }\end{array}$ & $\begin{array}{l}\text { Más } \\
\text { horas } \\
\text { sesión }\end{array}$ & $\begin{array}{c}\text { Mayor } \\
\text { Nivel } \\
\text { estudios }\end{array}$ & $\begin{array}{l}\text { Activ. } \\
\text { laboral } \\
\text { cualifi- } \\
\text { cada }\end{array}$ & $\begin{array}{l}\text { Problemas } \\
\text { Activid. } \\
\text { Cotidianas } \\
\text { (EQ-5D) }\end{array}$ & $\begin{array}{l}\text { Más } \\
\text { Dolor/ } \\
\text { Malestar } \\
\text { (EQ-5D) }\end{array}$ & $\begin{array}{c}\text { Más } \\
\text { Ansiedad/ } \\
\text { Depresión } \\
\text { (EQ-5D) }\end{array}$ \\
\hline Apariencia del personal & $-0,304$ & & & $-0,346$ & & & \\
\hline Personal cumple lo que dice & & & & $-0,340$ & & & \\
\hline Apariencia de la unidad & $-0,454$ & $-0,372$ & & & & & \\
\hline Información de los médicos & & & $-0,373$ & & & & $-0,409$ \\
\hline Tiempo de espera por médico & & & $-0,307$ & $-0,357$ & & & $-0,342$ \\
\hline Interés del personal en problemas & $-0,316$ & & & & & & \\
\hline Puntualidad de las sesiones & $-0,514$ & $-0,511$ & $-0,290$ & $-0,327$ & & & \\
\hline Rapidez en conseguir algo & $-0,346$ & $-0,305$ & & & & & \\
\hline Disposición del personal & $-0,326$ & $-0,290$ & & $-0,285$ & & & \\
\hline Capacidad del personal para comprender necesidades & & & & $-0,424$ & & & \\
\hline Información de medicación & & & $-0,290$ & & & & \\
\hline Información dieta enfermedad & & & $-0,286$ & & & & \\
\hline Amabilidad del personal & & & & & $-0,289$ & $-0,284$ & $-0,401$ \\
\hline Trato personalizado al paciente & & & & & $-0,330$ & & $-0,283$ \\
\hline
\end{tabular}

Tabla 2. Variables que disminuyen la Satisfacción 
En cuanto a las variables que aumentan la satisfacción, se encontraron correlaciones de Spearman estadísticamente significativas $(p<0,05)$ entre mayor edad del paciente y mayor satisfacción con la información que daban los médicos $(r=0,374)$; entre mejor estado de salud general medido por la Tarifa del EQ-5d y mayor satisfacción en la amabilidad del personal $(r=0,321)$; y entre que el paciente haga una mejor autovaloración de sí mismo con el Termómetro del EQ-5D y mayor satisfacción con la información que reciben de la dieta para la enfermedad renal. Por otro lado, mediante el Test de Student para comparar muestras independientes, se encontraron diferencias estadísticamente significativas $(p<0,05)$ en varias características del paciente, así aquellos que no sabían el nombre del médico, que creían que se les había explicado bien la enfermedad y la diálisis, que estaban bien atendidos y que su opinión era tenida en cuenta, que se consideraban satisfechos con el transporte, que habían elegido el tipo de diálisis, que nunca se habían trasplantado y que se dializaban por fístula estaban más satisfechos (tabla 3 ).

\begin{tabular}{|c|c|c|c|c|c|c|c|c|c|}
\hline & \multirow{2}{*}{$\begin{array}{c}\text { No saber } \\
\text { nombre } \\
\text { médico }\end{array}$} & \multicolumn{2}{|c|}{ Explicó bien } & \multirow{2}{*}{$\begin{array}{l}\text { Satisf. } \\
\text { Trans- } \\
\text { porte }\end{array}$} & \multirow{2}{*}{$\begin{array}{l}\text { Bien } \\
\text { aten- } \\
\text { dido }\end{array}$} & \multirow{2}{*}{$\begin{array}{l}\text { Fís- } \\
\text { tula }\end{array}$} & \multirow{2}{*}{$\begin{array}{c}\text { Eligió } \\
\text { TSR }\end{array}$} & \multirow{2}{*}{$\begin{array}{l}\text { Lo que } \\
\text { opina } \\
\text { cuenta }\end{array}$} & \multirow{2}{*}{$\begin{array}{c}\text { Nunca } \\
\text { Tras- } \\
\text { plante }\end{array}$} \\
\hline & & Enfer & diálisis & & & & & & \\
\hline Tecnología equipos médicos & & & & & & 0,04 & & & \\
\hline Tiempo de espera por médico & & & & & 0,01 & & & & \\
\hline Facilidad para llegar & & & & 0,001 & & & & & \\
\hline Interés del personal en los problemas. & 0,04 & 0,043 & & & & & 0,015 & & \\
\hline Preparación personal & 0,03 & & & & & & & & \\
\hline Personal comprende necesidades & 0,049 & & & & & & & & \\
\hline Comunicación del personal con familia & & 0,049 & 0,002 & & & & & & \\
\hline Interés de enfermería por pacientes & & & & & 0,01 & & 0,009 & 0,044 & \\
\hline Información de medicación & & & & 0,031 & & & & & \\
\hline Información dieta & & & 0,002 & & & & & & 0,047 \\
\hline Apariencia de la unidad & & & & & & & 0,047 & & \\
\hline Puntualidad de las sesiones & & & & & & & 0,035 & & 0,017 \\
\hline Disposición del personal & & & & & & & 0,005 & & \\
\hline Confianza que transmite el personal. & & & & & & & 0,037 & 0,038 & 0,03 \\
\hline Amabilidad del personal & & & & & & & 0,016 & 0,036 & \\
\hline Trato personalizado al paciente & & & & & & & 0,022 & & \\
\hline
\end{tabular}

Tabla 3. Variables que aumentan la Satisfacción

\section{Discusión-Conclusiones}

En los países desarrollados, los pacientes no son los mismos que hace unos años. La innovación tecnológica y los sistemas de información han hecho que los ciudadanos, potenciales enfermos, cada vez estén más informados sobre los servicios sanitarios, reclamen una atención más personalizada, más íntima y confidencial, conozcan más sus derechos, y tengan mayores expectativas como centro del sistema sanitario que son. Los cuidados que reciben los pacientes de la Unidad de HD, cumplen con las expectativas que los pacientes tienen de una Unidad de HD. En el presente trabajo se constata como los pacientes más jóvenes y aquellos con mayores niveles de estudios y que desarrollaron una actividad laboral más cualificada, tienen unas mayores expectativas de los servicios sanitarios y por lo tanto, son más exigentes.

Los niveles de satisfacción aquí encontrados son similares a los encontrados en otras unidades de España. Sin embargo, en nuestro estudio, sí se encontraron factores que se asociaban, aunque de forma baja o moderada, debido al tamaño de la muestra, a los distintos aspectos evaluados de la satisfacción. Así vemos que en función de la edad, del nivel de estudios alcanzado y el tipo de actividad laboral los pacientes juzgan la unidad con un nivel diferente de expectativas. $Y$ es que el concepto de satisfacción en la salud requiere el abandono del modelo paternalista, ya que la per- 
cepción de la autonomía por parte del paciente condiciona la satisfacción. Los pacientes que han podido elegir la terapia, que creen que su opinión es tenida en cuenta, que consideran que se les explica suficientemente la enfermedad y la terapia y que están correctamente atendidos, son los más satisfechos. Además el punto donde los pacientes están menos satisfechos es el relativo a la información que les proporcionan los médicos, mientras la mayor satisfacción la encuentran en la amabilidad y disposición del personal de enfermería. Un dato sorprendente es la mayor satisfacción de aquellos que no conocen el nombre del médico de la unidad, pero probablemente se debe a que son los que mejor estado de salud tienen.

A su vez existen factores propios de la enfermedad renal que condicionan la satisfacción. Con respecto a la terapia que reciben habría que considerar que los que se dializaban por fístula estaban más satisfechos con la tecnología y los equipos médicos y aquellos que ya habían estado trasplantados, que llevaban más tiempo en HD y que tenían sesiones más largas se mostraban menos, quizás por la mayor sobrecarga que les proporciona la diálisis, sobre todo a aquellos que disfrutaron de un trasplante, que es la terapia sustitutiva renal que más CVRS proporciona. Y es que, como vemos en el estudio, los que perciben más problemas para realizar las Actividades Cotidianas, más Dolor 0 Malestar y mayores niveles de Ansiedad/ Depresión tienen menos niveles de satisfacción. Creemos por todo ello importante el identificar los condicionantes, tanto relacionados con los tratamientos, como los que tienen que ver con la historia de la enfermedad, experiencias pasadas, duración, etc. como las variables inherentes al paciente, CVRS, estado de salud, etc. que están condicionando los niveles de satisfacción que reportan los pacientes.

\section{Agradecimientos}

Los autores están agradecidos a todos los pacientes que han participado en el estudio.

\section{Bibliografía}

1. Mira JJ, Lorenzo S, Rodríguez-Marín J, Aranaz J, Sitges E. La aplicación del modelo europeo de gestión de la calidad total al sector sanitario: ventajas y limitaciones. Rev Calidad Asistencial 1998;13: 9297.
2. Thompson AG, Suñol R. Expectations as determinants of patient satisfaction: concepts, theory and evidence. Int J Qual Health Care 1995; 7:127-141.

3. Coulter A. Evidence based patient information. BMJ 1998; 317: 225-226.

4. Levinson W, Gorawara-Bhat R, Dueck R, Egener B, Kao A, Kerr $C$, et al. Resolving disagreements in the patient-physician relationship. JAMA 1999; 282: 1477-1483.

5. Negro JM, Ramírez M, Toledo RF, Martínez R, Mérida C. Satisfacción de los pacientes asistidos en una consulta de alergología. Alergol Inmunol Clin 2004; 19:145-152.

6. Hernández ME, Ochando A, Mora J, Lorenzo S, López K. Satisfacción del paciente en una unidad de hemodiálisis: objetivo de calidad asistencial en enfermería. Rev Soc Esp Enferm Nefrol 2005; 8:90-96.

7. Hernández-Meca ME, Ochando-García A, Lorenzo Martínez S, López-Revuelta K. Factores determinantes de la satisfacción del paciente en tratamiento renal sustitutivo. Rev Soc Esp Enferm Nefrol. 2007; 10:6-13.

8. Cerro López $P$, Matani-Chugani V, Santos Ruiz AC. ¿Qué opinan los pacientes de diálisis del hospital Universitario NTRA. SRA. DE CANDELARIA sobre los servicios recibidos? Valoración de la calidad percibida NURE Inv [Revista en Internet] 2008 Marzo-Abril. 5(33). Disponible en: http://www.nureinvestigacion.es

9. Badia X, Roset M, Montserrat S, Herdman M, Segura A. La versión española del EuroQol: descripción y aplicaciones. Med Clin 1999; 112 (Supl. 1): 79-86.

10. Baztán JJ, Pérez del Molino J, Alarcón T, San Cristobal E, Izquierdo G, Manzarbeitia J. Índice de Barthel: instrumento válido para la valoración funcional de pacientes con enfermedad cerebrovascular, Rev Esp Geriatr y Gerontol 1993; 28: 32-40.

11. Karnofsky DA, Burchenal JHK: The clinical evaluation of chemotherapeutic agents in cancer. En: Evaluation of chemotherapeutic agents, edited by McLeod CM, Columbia University Press, New York 1948:191-205. 